\title{
Intravenous Ibuprofen 400 Versus 800 mg Every 6 Hours in Abdominal and Orthopedic Surgery: A Multicenter, Randomized Controlled Trial
}

\author{
Hong Zhang \\ Peking University People's Hospital \\ Ji-Han Huang
}

Shanghai University of Traditional Chinese Medicine

\section{Hong-Meng Xu}

The Fourth Hospital of Hebei Medical University

\section{Li Chen}

Second Hospital of Shanxi Medical University

\section{Hong Zheng}

First Affiliated Hospital of Xinjiang Medical University

\section{Zheng-Hua Hong}

Zhejiang Taizhou Hospital

\section{Lei Chen}

First Affiliated Hospital of Wenzhou Medical University

\section{Xi-Zhe Zhang}

Chifeng Municipal Hospital, Chifeng Clinical Medical School of Inner Mongolia Medical University

\section{Huai-Ping Liu}

Second Affiliated Hospital of Shantou University Medical College

\section{Chao-Yu Li}

Second People's Hospital of Neijiang

Qiu-Ye Lu

Meihekou City Central Hospital

\section{Yu-Jiang Bai}

Third Affiliated Hospital of Qiqihar Medical University

\section{Zu-Rong Hu}

Guangdong Women's and Children's Hospital

\section{Ya-Kui Zhang}

Beijing Luhe Hospital Affiliated to Capital Medical University

\section{Yong-Sheng Ma}

Tonghua Central Hospital,

\section{Xu-Yan Zhou}

First Hospital of Jiaxing

Yi Feng ( $\square$ doctor_yifeng@sina.com ) 


\section{Research Article}

Keywords: ibuprofen, pain, analgesic, nonsteroidal anti-inflammatory drug

Posted Date: September 8th, 2021

DOI: https://doi.org/10.21203/rs.3.rs-799074/v1

License: (c) (1) This work is licensed under a Creative Commons Attribution 4.0 International License. Read Full License 


\section{Abstract}

Background: Multimodal postoperative analgesia with intravenous ibuprofen can reduce the consumption of opioids and reduce the postoperative pain intensity. Recommended dose in the USA and Europe is $800 \mathrm{mg}$ every 6 hours for the management of moderate to severe postoperative pain. The purpose of this study was to investigate whether $800 \mathrm{mg}$ is needed in the Chinese population.

Methods: This is a multicenter, randomized, controlled multi-center trial. Adult patients (18-65 years of age) scheduled for abdominal or orthopedic surgery under general anesthesia with endotracheal intubation were eligible. Subjects were randomized at a ratio of 1:1:1 to receive ibuprofen at 400 or $800 \mathrm{mg}$ or saline every 6 hours for 48 hours after the operation. All subjects received morphine access through patient-controlled intravenous analgesia (PCIA) device, which was set as background infusion rate $0.5 \mathrm{mg} / \mathrm{h}$, bolus $1 \mathrm{mg}, 5$ minute lock interval and 20-mg 4-hour limit. The primary endpoint was morphine consumption within the first 24 hours after surgery.

Results: A total of 396 patients were enrolled ( 180 men, 216 women; $46 \pm 12.4$ years of age; 106 patients receiving abdominal surgery, and 290 patients receiving orthopedic surgery). The number of patients was 131 in the placebo group, 132 in the 400-mg group, and 133 in the 800-mg group. The 3 groups did not differ in age, sex, BMI and the type of surgery. Median 24-hour morphine consumption was $39.1 \mathrm{mg}$ (IQR: 29.7,61.7) $\mathrm{mg}$ in the placebo control, $29.8 \mathrm{mg}$ (IQR: 24.3,43.7) $\mathrm{mg}$ in the 400-mg group, and $28.0 \mathrm{mg}$ (IQR: 24.1, 35.9) in the 800-mg group ( $P<0.001$ for comparisons between placebo versus 400 and $800 \mathrm{mg}$ ibuprofen). There was no significant difference between the two ibuprofen groups $(P=0.125)$. Analgesia-related adverse events did not differ among the 3 groups.

Conclusions: Intravenous ibuprofen at either 400 or 800 mg every 6 hours could reduce postoperative morphine consumption, with apparently equal efficacy in Chinese population.

\section{Introduction}

Postoperative pain delays patient recovery and is associated with long-term psychological and mental harm $[1,2]$. Multimodal analgesia has been recommended by all major Guidelines, and non-steroidal antiinflammatory drugs (NSAIDs) play an important role in multimodal postoperative pain [3.4]. Ibuprofen is a classic NSAID with few adverse reactions [5]. The US FDA first approved the ibuprofen injection for the management of mild to moderate pain, moderate to severe pain in conjunction with narcotics, and for the reduction of fever in adults in 2009 [6, 7]. A previous trial by Southworth and colleagues [8] showed that intravenous ibuprofen at $800 \mathrm{mg}$ every 6 hours, but not at $400 \mathrm{mg}$, was effective in the management of postoperative pain. Whether $800 \mathrm{mg}$ is needed in the Chinese population is unknown.

\section{Methods}

This is a multicenter, randomized, controlled trial. The study protocol was approved by the Ethics Committee of Peking University People's Hospital (No. 2017PHA019) and all 14 participating centers. The study was conducted in accordance with the Declaration of Helsinki and Good Clinical Practice guidelines. All participants provided written informed consent. 


\section{Participants}

Adult patients (18-65 years of age) scheduled for abdominal surgery (intestinal or lower abdomen) or orthopedic surgery (e.g., knee arthroplasty and shoulder reconstruction) under general anesthesia with endotracheal intubation were eligible. Exclusion criteria included: 1) American Society of Anesthesiologists (ASA) Class III or higher; 2) body mass index (BMI) $<18$ or $>30 \mathrm{~kg} / \mathrm{m}^{2} ; 3$ ) inability to understand the NRS score; 4) history of allergy or hypersensitivity to ibuprofen, aspirin, NSAIDs, or any cyclooxygenase-2 inhibitors; 5) anemia; 6) pregnancy or lactation; 7) history of head trauma, intracranial surgery or stroke, or organic diseases of the central nervous system within 4 weeks before surgery; 8 ) history of congenital bleeding diseases (such as hemophilia), thrombocytopenia (platelet count below $80 \times 10^{9} / \mathrm{L}$ ), coagulation disorders; 9) history of organ transplantation or severe cardiovascular and cerebrovascular diseases, liver dysfunction (alanine aminotransferase or glutamic oxalacetic transaminase > 1.5 times normal upper limit), renal dysfunction (blood urea nitrogen > 1.5 times normal upper limit, creatinine > normal upper limit), endocrine system and mental illness; 10) history of gastrointestinal ulcer or bleeding; 11) receiving warfarin, lithium, methotrexate, clopidogrel or other long-term anticoagulant or anti-platelet drugs, two or more antihypertensive agents; 12) exposure to any analgesic, muscle relaxant, or sedative within 24 hours.

\section{Study Medications}

Ibuprofen (400 mg in 4-ml; batch \#: 170101/181103) and placebo (saline, 4-ml; batch \#:170102/181001, the specification of the placebo, such as appearance, color, and odor, was consistent with ibuprofen injection) were provided by Hainan Herui Pharmaceutical Company (Hainan, China). Each package of medication for each subject contained 9 doses with same combination: 2 ampoules of ibuprofen (800mg ibuprofen), or 1 ampoule of ibuprofen and 1 ampoule of saline (400mg ibuprofen), or 2 ampoules of saline (placebo) for per dose.

\section{Randomization and blinding}

The three kinds of study drug packages were randomly assigned in a ratio of 1:1:1 using the computergenerated random number sequence. Based on the allocation sequence, drug packages were labeled serially in pharmaceutical company. Subjects were randomized to be assigned to one of the three groups in the order of recruitment and received intravenous infusion of placebo, ibuprofen 400mg or ibuprofen $800 \mathrm{mg}$. The researchers, patients and doctors, were all blinded throughout the study. The complete random sequence was kept by the primary researcher in Peking University People's Hospital. Emergency envelopes containing the random allocation individually were prepared for each center, which could be unsealed only in the case of serious adverse events.

\section{Study Design}

Subjects were received intravenous infusion of placebo, or ibuprofen at 400 or $800 \mathrm{mg}$ every 6 hours for total of 9 doses, from the time of wound closure to 48 hours. Each infusion lasted for $30 \mathrm{~min}$. All subjects received 5-mg intravenous morphine at the time of wound closure and PCIA (Fig. 1). For PCIA, 100-mg morphine was dissolved in 200-ml saline, the background infusion rate was $0.5 \mathrm{mg} / \mathrm{h}$, bolus was $1 \mathrm{mg}$, with a lockout time of 5 min and 20-mg 4-hour limit. Subjects had access to morphine via PCIA throughout the treatment period. 
Study protocol mandated discontinuation of study medication if the patient required the use of additional pain medication, including a narcotic other than morphine or any other non-narcotic pain medication including NSAIDs due to the inability to manage pain by PCIA. These cases were classified as treatment failures.

Anesthesia was induced using 0.03-mg/kg midazolam, $1.5 \sim 2.5-\mathrm{mg} / \mathrm{kg}$ propofol or 0.3-mg/kg etomidate, 3$\mu \mathrm{g} / \mathrm{kg}$ fentanyl, and $0.6-\mathrm{mg} / \mathrm{kg}$ rocuronium or $0.1-\mathrm{mg} / \mathrm{kg}$ vecuronium. Anesthesia was maintained using propofol and remifentanil, or sevoflurane inhalation and remifentanil. It was allowed to add muscle relaxants according to the need of operation and add fentanyl $1 \mu \mathrm{g} / \mathrm{kg}$ every 2 hours after anesthesia induction (not allowed within 1.5 hours before the end of the operation). Neuraxial anesthesia and regional blockade were not permitted. According to the study protocol, dexmedetomidine, sufentanil, tramadol, penehyclidine hydrochloride, and scopolamine were not permitted during the surgery; aspirin, other NSAIDs and other drugs with antipyretic (e.g., glucocorticoids) and analgesic effects or anticoagulants (except low molecular weight heparin, LMWH), thrombolytic drugs (streptokinase, urokinase), angiotensin-converting enzyme inhibitors (ACEI), diuretics, lithium agents, methotrexate, etc., were prohibited during the study period.

\section{Outcomes}

The primary endpoint measure was morphine consumption within the first 24 hours after surgery, calculated according to the intent-to-treat principle. The secondary endpoints included: 1) Pain intensity at rest and with movement. Pain intensity was evaluated using patient self-reporting with numerical rating scales (NRS) $(0=$ no pain to $10=$ intense pain) at $1,2,3,6,12,18,24,30,36,42$ and 48 hours after the first administration of test drugs. In patients who were asleep at the time of evaluation, the pain score was recorded as 2. 2) The area under the pain intensity-time curve (AUC) both at rest and with movement during 1-24 hours after surgery. 3) The number of total and effective PCIA pushes within the first 24 hours after surgery. 4) Percentage of the treatment failures. 5) Incidence of pyrexia (defined body temperature $\geq 38^{\circ} \mathrm{C}$ ) during the study period. 6) Analgesia-related adverse events and laboratory assessments (included biochemistry, electrolytes, blood routine, blood coagulation function, urinalysis, and electrocardiogram) the third day after surgery.

\section{Statistical Analysis}

Sample size calculation was based on the following assumptions: 1) 24-hour morphine consumption at 55.9 $\mathrm{mg}(\mathrm{SD}=20.6)$ in the placebo group vs. $47.3 \mathrm{mg}(\mathrm{SD}=25.6)$ in the 800-mg group [13]; 2) 2-sided a at 0.05 and $1-\mathrm{b}$ at 0.8 . The calculation yielded 116 subjects in each group. Assuming $20 \%$ dropout rate, we planned to enroll 396 patients.

Continuous variables were presented as the mean \pm standard deviation (SD) if following normal distribution, and as median (interquartile range; IQR) otherwise. The 24-hour morphine consumption was analyzed using ANCOVA after Box-Cox transformation, with intervention, center, patient age, and weight as covariates. Pain scores were analyzed using a mixed effect model for repeated measurement. Area under the pain intensitytime curve (AUC) was compared among groups using Kruskal-Wallis and Nemenyi tests. Chi-square test was used to analyze categorical variables. $\mathrm{P}<0.05$ was considered statistically significant. All statistical analyses were conducted using SAS 9.4. 


\section{Results}

A total of 465 patients were screened, and 396 participants were eligible. 396 participants (131 to placebo control, 132 to 400 -mg group and 133 to $80-\mathrm{mg}$ group) were randomized and received at least a partial dose of IV-ibuprofen or placebo and were included in the ITT population. Among the 396 subjects, 38 did not receive all 4 planned doses for a variety of reasons: 5 for consent withdrawal, 12 for violation of the protocol (e.g., using banned drugs during or after surgery, the method of anesthesia or anesthetics not conform to the protocol) and 21 patients due to side effects such as dizziness, drowsiness, nausea, vomiting, pruritus. The remaining 358 patients completed the first 4 doses of study medication according to pre-planned protocol (during the 24-hour time frame of the primary end point) and were included in the PP population (119 to placebo control, 118 to 400-mg group and 121 to 80-mg group) (Fig. 2).

Baseline demographic and the type of surgery did not differ among the 3 groups (Table 1).

Table 1. Baseline characteristics of the patients.

\begin{tabular}{|lllll|}
\hline & $\begin{array}{l}\text { Placebo } \\
\mathrm{n}=131\end{array}$ & 400 -mg ibuprofen $\mathrm{n}=132$ & 800 -mg ibuprofen $\mathrm{n}=133$ & $\mathrm{P}$ \\
\hline Age $(\mathrm{y})$, mean $\pm \mathrm{SD}$ & $47 \pm 12.8$ & $45 \pm 12.6$ & $46 \pm 11.8$ & 0.44 \\
\hline Male sex, $\mathrm{n}(\%)$ & $50(38.2)$ & $60(45.5)$ & $70(52.6)$ & 0.06 \\
\hline Weight $(\mathrm{kg})$, mean $\pm \mathrm{SD}$ & $65.7 \pm 10.4$ & $63.6 \pm 10.3$ & $65.7 \pm 11.5$ & 0.20 \\
\hline BMI $\left(\mathrm{kg} / \mathrm{m}^{2}\right)$, mean $\pm \mathrm{SD}$ & $24.3 \pm 2.7$ & $23.6 \pm 2.9$ & $24.0 \pm 2.9$ & 0.18 \\
\hline Type of surgery, $\mathrm{n}(\%)$ & & & & 0.72 \\
\hline Abdominal & $33(25.2)$ & $35(26.5)$ & $35(26.3)$ & \\
\hline Orthopedic & $98(74.8)$ & $97(73.5)$ & $98(73.7)$ & \\
\hline
\end{tabular}

In the ITT population analysis, median 24-hour morphine consumption was 39.1 mg (IQR: 29.7, 61.7) in the placebo control, $29.8 \mathrm{mg}$ (IQR: 24.3, 43.7) in the 400-mg group, and $28.0 \mathrm{mg}$ (IQR: 24.1, 35.9) in the 800mg group ( $\mathrm{P}<0.001$ for comparisons between placebo versus both ibuprofen at both doses) (Table 2). There was no significant difference between the two ibuprofen groups $(P=0.125)$. The results of the PP population analysis were consistent, median 24-hour morphine consumption was $38.4 \mathrm{mg}(\mathrm{IQR}: 29.7,57.8)$ in the placebo control, $29.8 \mathrm{mg}$ (IQR: 24.3, 43.6) in the 400-mg group, and $28.0 \mathrm{mg}$ (IQR: 24.1, 35.8) in the 800-mg group $(\mathrm{P}<0.001$ for comparisons between placebo versus both ibuprofen dosage) (Table 2).

Table 2. The total amount of morphine during the first 24 hours

*Least squares (LS) means were adjusted for age group, weight group, randomization center, and study group.

†Versus placebo. ¥Versus ibuprofen 400mg group 


\begin{tabular}{|c|c|c|c|c|}
\hline & Placebo control & 400-mg ibuprofen & 800-mg ibuprofen & \\
\hline \multicolumn{4}{|l|}{ ITT population } & scores \\
\hline$n$ & 131 & 132 & 133 & both at \\
\hline \multicolumn{4}{|c|}{ Amount of Morphine (mg) } & rest and \\
\hline Mean \pm SD & $51.41 \pm 32.48$ & $38.47 \pm 23.94$ & $36.21 \pm 23.88$ & \\
\hline Median (Q1, Q3) & $39.10(29.70,61.70)$ & $29.75(24.30,43.70)$ & $28.00(24.10,35.90)$ & \\
\hline \multicolumn{4}{|c|}{ Transformed Amount of morphine (Box-Cox) } & \\
\hline LS Mean (SE)* & $3.78(0.04)$ & $3.54(0.04)$ & $3.45(0.04)$ & \\
\hline \multirow[t]{2}{*}{ P } & & $\nabla 0.001 \dagger$ & $\nabla 0.001 \dagger$ & \\
\hline & & & $0.125 \ddagger$ & \\
\hline \multicolumn{4}{|l|}{ PP population } & \\
\hline$n$ & 119 & 118 & 121 & \\
\hline \multicolumn{4}{|c|}{ Amount of Morphine (mg) } & \\
\hline Mean \pm SD & $48.63 \pm 28.23$ & $39.24 \pm 24.52$ & $35.01 \pm 18.56$ & \\
\hline Median (Q1, Q3) & $38.40(29.70,57.80)$ & $29.80(24.30,43.60)$ & $28.00(24.10,35.80)$ & \\
\hline \multicolumn{4}{|c|}{ Transformed Amount of morphine (Box-Cox) } & \\
\hline LS Mean (SE)* & $3.76(0.04)$ & $3.55(0.04)$ & $3.47(0.04)$ & \\
\hline \multirow[t]{2}{*}{ P } & - & $\nabla 0.001 \dagger$ & $\otimes 0.001 \dagger$ & \\
\hline & - & - & $0.173 \ddagger$ & \\
\hline
\end{tabular}

movement differed significantly among the 3 groups $(P<0.05)$, with the highest in the placebo control, and the lowest in the 800-mg group (Figures 3 and 4).

The pain intensity-time AUC was different among three groups both at rest and with movement during 1-24 hours after surgery $(P<0.001)$. It was greater in the placebo group than in the 2 ibuprofen groups both at rest ( $P<0.001$ for 800-mg ibuprofen group, $P=0.003$ for 400-mg ibuprofen group) and with movement $(P<0.001$ for 800 -mg ibuprofen group, $P=0.026$ for $400-\mathrm{mg}$ ibuprofen group). There was no difference between the $400-\mathrm{mg}$ and the 800-mg groups at rest $(P=0.386)$ and with movement $(P=0.131)$ (Table 3$)$.

Table 3. Pain intensity-time AUC (1-24h) at rest and with movement 


\begin{tabular}{|c|c|c|c|c|c|c|}
\hline & \multirow{2}{*}{$\begin{array}{l}\text { Placebo } \\
\text { control }(n=131)\end{array}$} & \multirow{2}{*}{$\begin{array}{l}\text { 400-mg } \\
\text { ibuprofen ( } n=132)\end{array}$} & \multirow{2}{*}{$\begin{array}{l}\text { 800-mg } \\
\text { ibuprofen ( } n=133)\end{array}$} & \multicolumn{3}{|l|}{$P$} \\
\hline & & & & $\begin{array}{l}400-\mathrm{mg} \\
V s \\
\text { Placebo }\end{array}$ & $\begin{array}{l}800-\mathrm{mg} \\
\text { Vs } \\
\text { Placebo }\end{array}$ & $\begin{array}{l}800-\mathrm{mg} \\
v s 400- \\
\mathrm{mg}\end{array}$ \\
\hline \multicolumn{7}{|l|}{ At rest } \\
\hline mean $\pm S D$ & $53.65 \pm 23.658$ & $44.87 \pm 21.116$ & $41.09 \pm 22.439$ & 0.026 & $<0.001$ & 0.386 \\
\hline \multicolumn{7}{|c|}{ With movement } \\
\hline mean $\pm S D$ & $75.54 \pm 25.783$ & $65.09 \pm 23.100$ & $58.68 \pm 23.885$ & 0.003 & $<0.001$ & 0.131 \\
\hline
\end{tabular}

The number of total PCIA pushes during the first 24 hours after surgery was $28.5 \pm 48.4$ in the placebo control, $28.1 \pm 113.7$ in the 400 -mg group, and $17.2 \pm 58.5$ in the 800 -mg group (17.2 \pm 58.5$)(P=0.422)$. However, the number of effective PCIA pushes was higher in the placebo group $(13.6 \pm 15.8)$ than in the 400 -mg group $(8.5 \pm 14.9, P=0.003)$ and the $800-m g$ group $(6.1 \pm 10.2, P<0.001)$. There was no difference in the two ibuprofen groups $(P=0.158)$.

Percentage of the treatment failures was 3.8\% (5/131) in the placebo control, 2.3\% (3/132) in the 400-mg group and $1.5 \%(2 / 133)$ in the $800-\mathrm{mg}$ group. There was no difference among three groups. $(P=0.432)$.

The rate of pyrexia was different among three groups $(P<0.001)$ (Table 4), 30.5\% $(40 / 131)$ in the placebo control, $19.7 \%(26 / 132)$ in the $400-\mathrm{mg}$ group ( $P=0.128 \mathrm{vs}$. the placebo control) and $8.3 \%(11 / 133)$ in the $800-$ $\mathrm{mg}$ group ( $\mathrm{P}<0.001 \mathrm{vs}$. the placebo control, $P=0.022 \mathrm{vs.}$ the $400-\mathrm{mg}$ group).

There was no gastrointestinal bleeding or postoperative surgical site bleeding that required transfusion during the study. Cardiovascular thrombotic events, anaphylactoid reactions, and bronchospasm had not observed during the study period. The laboratory examination on the third day after the surgery showed no renal dysfunction (blood urea nitrogen $>1.5$ times normal upper limit, creatinine $>$ normal upper limit).

The incidence of hepatic enzymes elevated (one or both of alanine aminotransferase or glutamic oxalacetic transaminase $>1.5$ times normal upper limit) was $2.3 \%(3 / 131)$ in the placebo control, $2.3 \%(3 / 132)$ in the 400-mg group and $0.8 \%(1 / 133)$ in the $800-\mathrm{mg}$ group. There was no difference among three groups $(P=0.55)$.

The incidence of common analgesia-related adverse events (including nausea and vomiting, dizziness, flatulence, constipation and pruritus) except for pain at the injection site was not increase in the ibuprofen groups than in the placebo group. (Table 4).

Table 4. Pyrexia and the common analgesia-related adverse events. 


\begin{tabular}{lllll} 
Event & $\begin{array}{l}\text { Placebo } \\
\text { control } \\
\mathrm{n}=131\end{array}$ & $\begin{array}{l}400-\mathrm{mg} \\
\text { ibuprofen } \mathrm{n}=132\end{array}$ & $\begin{array}{l}\text { 800-mg } \\
\text { ibuprofen } \mathrm{n}=133\end{array}$ & $\mathrm{P}$ \\
\cline { 2 - 3 } & $\mathrm{n}(\%)$ & $\mathrm{n}(\%)$ & $\mathrm{n}(\%)$ & $<0.001$ \\
\hline Pyrexia & $40(30.5)$ & $26(20.0)^{\dagger}$ & $11(8.3)^{\star}$ & 0.97 \\
\hline Nausea & $35(26.7)$ & $37(28.0)$ & $36(27.1)$ & 0.25 \\
\hline Vomit & $30(22.9)$ & $35(26.5)$ & $24(18.0)$ & 0.85 \\
\hline Dizziness & $11(8.4)$ & $9(6.8)$ & $9(6.8)$ & 0.73 \\
\hline Flatulence & $7(5.3)$ & $6(4.5)$ & $9(6.8)$ & 0.02 \\
\hline Constipation & $6(<0.1)$ & $4(<0.1)$ & $0(0)^{\#}$ & 0.44 \\
\hline Pruritus & $1(<0.1)$ & $2(<0.1)$ & $0(0)$ & $<0.001$
\end{tabular}

${ }^{*} P<0.001$, versus placebo. $+P=0.128$, versus placebo. $\ddagger P=0.007$, versus placebo. $\S P=0.014$, versus placebo.

\section{Discussion}

Ibuprofen is a classic NSAIDs, with definite anti-inflammatory, antipyretic, and analgesic effects. After the injection formulation was marketed in the United States, its clinical application became more and more widespread. In addition to the pain management in some difficult oral situations such as acute post-traumatic or renal colic pain control, many outpatient oral treatments (such as molar extraction [9]) and minor operations (such as tonsillectomy [10], laparoscopic cholecystectomy [11]) ibuprofen injection can play an effective antipyretic analgesic and anti-inflammatory effects. Ibuprofen has a better analgesic effect than acetaminophen alone, and it can have a better analgesic effect in combination with acetaminophen. Ibuprofen has a faster onset and better analgesic effect than Ketorolac [12], another intravenous NSAIDs drug.

In the treatment of moderate and severe postoperative pain, multimodal analgesia with ibuprofen injection reduces effectively the dosage of opioids and the postoperative pain intensity. The first randomized, doubleblind, placebo-controlled study of ibuprofen injection was conducted by Southworth SR et al in 2009 [8]. The 406 participants at 17 centers in the United States, Australia, and South Africa included Caucasian, Black, Asian and Hispanic and other different races. The study results showed that IV ibuprofen $800 \mathrm{mg}$ but not $400 \mathrm{mg}$ q6h in the management of postoperative pain was associated with a significant reduction in morphine consumption in the first 24 hours after surgery compared with placebo. In subsequent studies for moderate and severe postoperative pain, ibuprofen $800 \mathrm{mg}$ was mostly used. For example, in the study of Singla et al. [13] of postoperative analgesia for orthopedic surgery completed at eight hospitals, six in the United States and two in South Africa. Another multicenter study for abdominal and orthopedic surgery was conducted in Europe (Spain) [14]. Other studies [15-20] were conducted in Turkey, US and other different regions. None of these studies revealed that multimodal postoperative analgesia with IV ibuprofen $400 \mathrm{mg}$ q6h for moderate 
and severe pain was associated with reduction of morphine consumption. Multicenter study of ibuprofen injection for moderate and severe postoperative analgesia in Chinese population has not been reported so far.

Interestingly, in the present study, the administration of IV ibuprofen $400 \mathrm{mg}$ q6h compared with placebo in the management of moderate or severe postoperative pain was as effective as $800 \mathrm{mg}$, associated with a significant reduction in morphine consumption in the first 24 hours after surgery, and also associated with a significant reduction of pain intensity-time AUC either resting or motion after administration. These results suggested that IV-ibuprofen $400 \mathrm{mg}$ can be effectively used on Chinese populations with moderate and severe postoperative pain.

In some previous studies, IV-ibuprofen $800 \mathrm{mg}$ reduced morphine doses by $19-32 \%$ during the first $24 \mathrm{~h}$ after surgery. The reduction of morphine using IV-ibuprofen $800 \mathrm{mg}$ in this study was $27 \%$, which was consistent with previous results. Moreover, we found that IV-ibuprofen $400 \mathrm{mg}$ reduced morphine dosage by $22 \%$, which was also within the range from previous studies.

The difference in the weight of the participants from other studies is probably the main reason why $400 \mathrm{mg}$ was effective in this study. In the study of Southworth SR., the mean weight of participants was $83.8 \mathrm{~kg}$ (placebo group, $83.4 \mathrm{~kg} ; 400 \mathrm{mg}$ ibuprofen group $83.0 \mathrm{~kg}$; and $800 \mathrm{mg}$ ibuprofen group $84.9 \mathrm{~kg}$, respectively), which was higher than $65.0 \mathrm{~kg}$ in this study (placebo group, $65.7 \mathrm{~kg} ; 400 \mathrm{mg}$ ibuprofen group, $63.6 \mathrm{~kg}$; and $800 \mathrm{mg}$ ibuprofen group, $65.7 \mathrm{~kg}$, respectively). The efficacy of ibuprofen $400 \mathrm{mg}$ in reducing morphine consumption in a non-Chinese population with lower body weight is worth looking into for further study, in which clinical medication may require consideration of patients' weight.

A meta-analysis [21] published in 2015 comprised ten, Phase II-IV studies conducted between 2002 and 2014 with IV ibuprofen in adult patients. The adverse events incidence of IV ibuprofen was low compared to placebo, and dose adjustments were not required in the elderly population. Except pain at the site of infusion, there was no significant increase in perioperative cardiovascular, gastrointestinal, renal, hepatic or respiratory adverse events or surgical bleeding in patients received IV ibuprofen compared with patients who received IV placebo. This current study was consistent with these results. The pain at the infusion site was mild and no special treatment was required. The same conclusion was reached in previous studies on the pharmacokinetics and safety of ibuprofen injection in healthy Chinese volunteers [22]. Therefore, IV ibuprofen $400 \mathrm{mg}$ and $800 \mathrm{mg}$ can be safely used in Chinese population.

In addition to analgesic effects, ibuprofen use is associated with antipyretic and anti-inflammatory effects. Different from the previous study by Southworth et al. [8], in this study, the incidence of pyrexia only decreased statistically significantly in the ibuprofen $800 \mathrm{mg}$ group but not $400 \mathrm{mg}$, compared with the placebo group. This was indicated under the surgical model of this study, the antipyretic effect was dose-dependent. This point was consistent with the study conducted by Bookstaver P.B. et al. [7] that showed an increase percentage of patients with reduction of temperature following an increase in intravenous ibuprofen doses. The antipyretic effect of ibuprofen significantly reduces the incidence of pyrexia, thus increases the comfort of patients [23]. Both analgesia and antipyretic could increase patients' comfort during medication.

Limitations

Page 10/16 
The study has several limitations. Firstly, only two doses of ibuprofen were studied, which could not clarify the dose-response relationship of ibuprofen, and could not determine the optimal dose for moderate and severe postoperative analgesia. Secondly, this study did not involve more types of surgery than abdominal and orthopedic surgery. Thirdly, the actual individual pain intensity could not be obtained before administration, so it was impossible to conduct stratified research according to different post-operative pain degrees. In addition, resent studies have shown that preoperative single-dose intravenous ibuprofen can reduce postoperative pain and opioid consumption until $24 \mathrm{~h}$ postoperatively [24]. In this study, medication was infused intravenously from the time of wound closure. It is conceivable that morphine consumption may be more effectively reduced if medication was infused before the skin incision. As a result, further research is needed to confirm.

\section{Conclusion}

In patients undergoing abdominal or orthopedic surgery, IV-ibuprofen at either 400 or $800 \mathrm{mg}$ every 6 hours could reduce postoperative morphine consumption, with apparently equal efficacy in Chinese population. IVibuprofen $800 \mathrm{mg}$ is not needed in the management of postoperative moderate to severe pain in the Chinese population.

\section{Abbreviations}

ACEl: angiotensin-converting enzyme inhibitors; ANCOVA: analysis of covariance; ASA: American Society of Anesthesiologists; AUC: area under the pain intensity-time curve; BMI: body mass index; ICF: Informed Consent Form; IQR: interquartile range; ITT: intent-to-treat; LMWH: low molecular weight heparin; NRS: numeric rating scale; NSAIDs: non-steroidal anti-inflammatory drugs; PCIA: patient-controlled intravenous analgesia; PP: perprotocol; SD: standard deviation

\section{Declarations}

\section{Acknowledgements}

The authors would like to thank all study participants who were enrolled in this study.

\section{Funding}

This study received no specific funding.

\section{Availability of data and materials}

The datasets used and/or analysed during the current study are available from the corresponding author on reasonable request.

\section{Competing interests}

The authors declare that they have no competing interests.

\section{Ethics approval and consent to participate}


The study protocol was approved by the Ethics Committee of Peking University People's Hospital (No. 2017PHA019) and all 14 participating centers. All participants provided written informed consent.

\section{Consent for publication}

Not applicable.

\section{Authors' contributions}

Study conception: YF. Study design: all authors. Study protocol draft: YF and HZ. Study protocol revise: all authors. Study conduct and data collection: HZ, HMX, LC, HZH, ZHH, LCH, XZZ, HPL, CYL, QYL, YJB, ZRH, YKZ, YSM, and XYZ. Statistical analysis: JHH. Drafting of the manuscript: HZ. YF is the principal investigator and has overall responsibility for the trial. All authors have read and approved the final manuscript.

\section{References}

1. Rathmell JP, Wu CL, Sinatra RS, Ballantyne JC, Ginsberg B, Gordon DB, et al. Acute post-surgical pain management: a critical appraisal of current practice. Reg Anesth Pain Med. 2006;31:1-42. doi: 10.1016/j.rapm.2006.05.002.

2. Correll D. Chronic postoperative pain: recent findings in understanding and management. F1000Res. 2017 Jul 4;6:1054. doi: 10.12688/f1000research.11101.1. PMID: 28713565; PMCID: PMC5499782.

3. Desai K, Carroll I, Asch SM, Seto T, McDonald KM, Curtin C, et, al. Utilization and effectiveness of multimodal discharge analgesia for postoperative pain management. J Surg Res. 2018; 228:160-9. doi: 10.1016/j.jss.2018.03.029.

4. Chou R, Gordon DB, Oscar A, Rosenberg JM, Bickler S, Brennan T, et, al. Guidelines on the management of postoperative pain: management of postoperative pain: A clinical practice guideline from the American Pain Society, the American Society of Regional Anesthesia and Pain Medicine, and the American Society of Anesthesiologists' Committee on Regional Anesthesia, Executive Committee, and Administrative Council. The Journal of Pain. 2016; 17:131-57. doi: 10.1016/j.jpain.2015.12.008.

5. KD Rainsford. Discovery, mechanisms of action and safety of ibuprofen. Int J Clin Pract Suppl. 2003; 135:3-8.

6. Scott LJ. Intravenous ibuprofen: in adults for pain and fever. Drugs. 2012; 72:1099-109. doi: 10.2165/11209470-000000000-00000

7. Bookstaver P B, Miller A D, Rudisill C N, Norris LB. Intravenous ibuprofen: The first injectable product for the treatment of pain and fever. J Pain Res. 2010; 25:67-79. doi: 10.2147/jpr.s6993.

8. Southworth S, Peters J, Rock A, Pavliv L. A multicenter, randomized, double-blind, placebo-controlled trial of intravenous ibuprofen 400 and 800 mg every 6 hours in the management of postoperative pain. Clin Ther. 2009; 31(9):1922-35. doi: 10.1016/j.clinthera.2009.08.026.

9. Viswanath A, Oreadi D, Finkelman M, Klein G, Papageorge M. Does pre-emptive administration of intravenous ibuprofen (Caldolor) or intravenous acetaminophen (Ofirmev) reduce postoperative pain and subsequent narcotic consumption after third molar surgery?. J Oral Maxillofac Surg. 2019; 77:262-270. 2019;77(2):262 - 70. doi:10.1016/j.joms.2018.09.010 
10. Moss JR, Watcha MF, Bendel LP, McCarthy DL, Witham SL, Glover CD. A multicenter, randomized, doubleblind placebo-controlled, single dose trial of the safety and efficacy of intravenous ibuprofen for treatment of pain in pediatric patients undergoing tonsillectomy. Paediatr Anaesth. 2014; 24:483-9. doi:10.1111/pan.12381

11. Karaca O, Pınar HU, Turk E, Dogan R, Ahiskalioglu A, Solak SK. Effects of single-dose preemptive pregabalin and intravenous ibuprofen on postoperative opioid consumption and acute pain after laparoscopic cholecystectomy. J Invest Surg. 2019; 32:189-95. doi:10.1080/08941939.2017.1386738

12. Forouzanfar MM, Mohammadi K, Hashemi B, Safari S. Comparison of intravenous ibuprofen with intravenous ketorolac in renal colic pain management; a clinical trial. Anesth Pain Med. 2019; 9: e86963. doi:10.5812/aapm.86963.

13. Singla N, Rock A, Pavliv L. A multi-center, randomized, double-blind placebo-controlled trial of intravenous-ibuprofen (IV-ibuprofen) for treatment of pain in post-operative orthopedic adult patients. Pain Med. 2010; 11:1284-93. doi: 10.1111/j.1526-4637.2010.00896.

14. Gago Martínez A, Escontrela Rodriguez B, Planas Roca A, Martínez Ruiz A. Intravenous ibuprofen for treatment of post-operative pain: a multicenter, double blind, placebo-controlled, randomized clinical trial. PLoS One. 2016; 11: e0154004. doi: 10.1371/journal.pone.0154004.

15. Gürkan Yavuz,Yörükoğlu Hadi Ufuk,Işık Erdal,Kuş Alparslan. The effect of ibuprofen on postoperative opioid consumption following total hip replacement surgery. Turkish journal of anaesthesiology and reanimation. 2019; 47:31-34. doi: 10.5152/TJAR.2018.48265.

16. Peter B. Kroll, Laura Meadows, Amy Rock, Leo Pavliv. A multicenter, randomized, double-blind, placebocontrolled trial of intravenous ibuprofen (IV-ibuprofen) in the management of postoperative pain following abdominal hysterectomy. Pain Practice,2011; 11:23-32. doi: 10.1111/j.15332500.2010.00402.x

17. Onda A, Ogoshi A, Itoh M, Nakagawa T, Kimura M. Comparison of the effects of treatment with celecoxib, loxoprofen, and acetaminophen on postoperative acute pain after arthroscopic knee surgery: A randomized, parallel-group trial. J Orthop Sci. 2016; 21:172-7. doi: 10.1016/j.jos.2015.11.005.

18. Oreskovic Z, Bicanic G, Hrabac P, Tripkovic B, Delimar D. Treatment of postoperative pain after total hip arthroplasty: comparison between metamizol and paracetamol as adjunctive to opioid analgesicsprospective, double-blind, randomised study. Arch Orthop Trauma Surg. 2014; 134:631-6. doi: 10.1007/s00402-014-1979-7.

19. Gupta A, Abubaker H, Demas E, Ahrendtsen L. A randomized trial comparing the safety and efficacy of intravenous ibuprofen versus ibuprofen and acetaminophen in knee or hip arthroplasty. Pain Physician 2016; 19:349-56. PMID: 27454264.

20. Weisz RD, Fokin AA, Lerner V, Flynt A, Macias-Perez I, Pavliv L, et, al. Intravenous ibuprofen reduces opioid consumption during the initial 48 hours after injury in orthopedic trauma patients. J Orthop Trauma. 2020;34:341-7. doi: 10.1097/BOT.0000000000001733.

21. Southworth SR, Woodward EJ, Peng A, Rock AD. An integrated safety analysis of intravenous ibuprofen (Caldolor(ß)) in adults. J Pain Res 2015; 8:753-65. doi: 10.2147/JPR.S93547.

22. Zhou H, Xu W, Wu G, Wu L, Shentu J, Pan Z, et, al. Pharmacokinetics and tolerability of intravenous ibuprofen injection in healthy Chinese volunteers: a randomized, open-label, single- and multiple-dose 
study. Int J Clin Pharmacol Ther 2016; 54:904-13. doi: 10.5414/CP202603.

23. Barbagallo M, Sacerdote P. Ibuprofen in the treatment of children's inflammatory pain: a clinical and pharmacological overview. Minerva Pediatr. 2019;71:82-99. doi:10.23736/S0026-4946.18.05453-1.

24. Kim SY, Lee S, Lee Y, Kim H, Kim KM. Effect of single dose preoperative intravenous ibuprofen on postoperative pain and opioid consumption: a systematic review and meta-analysis. Korean J Anesthesiol. 2021 Feb 22. doi: 10.4097/kja.21050. Epub ahead of print. PMID: 33611881.

\section{Figures}

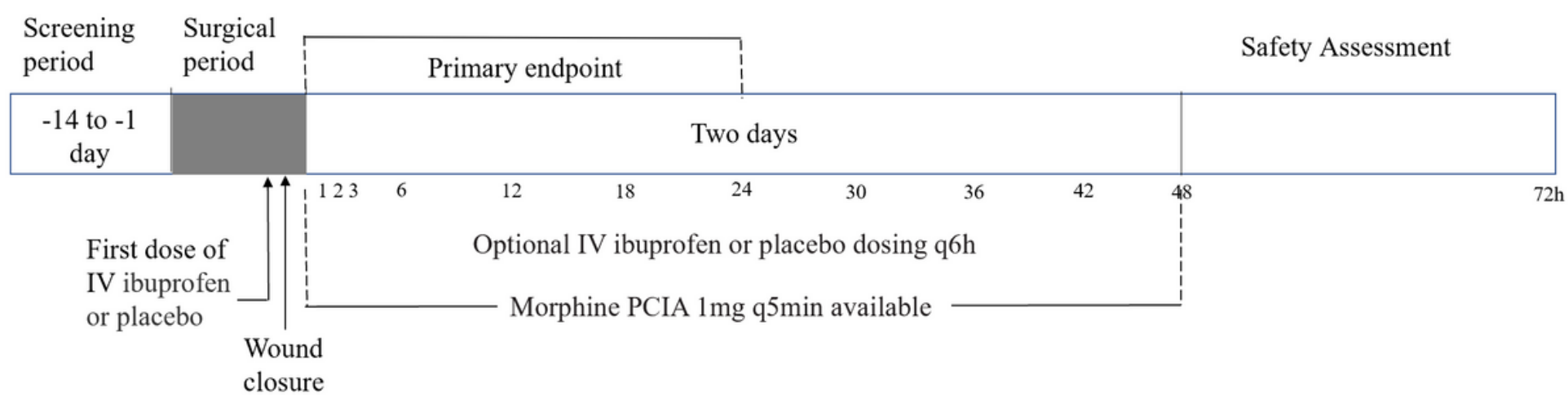

\section{Figure 1}

Study timeline. $\mathrm{PCIA}=$ patient-controlled intravenous analgesia.

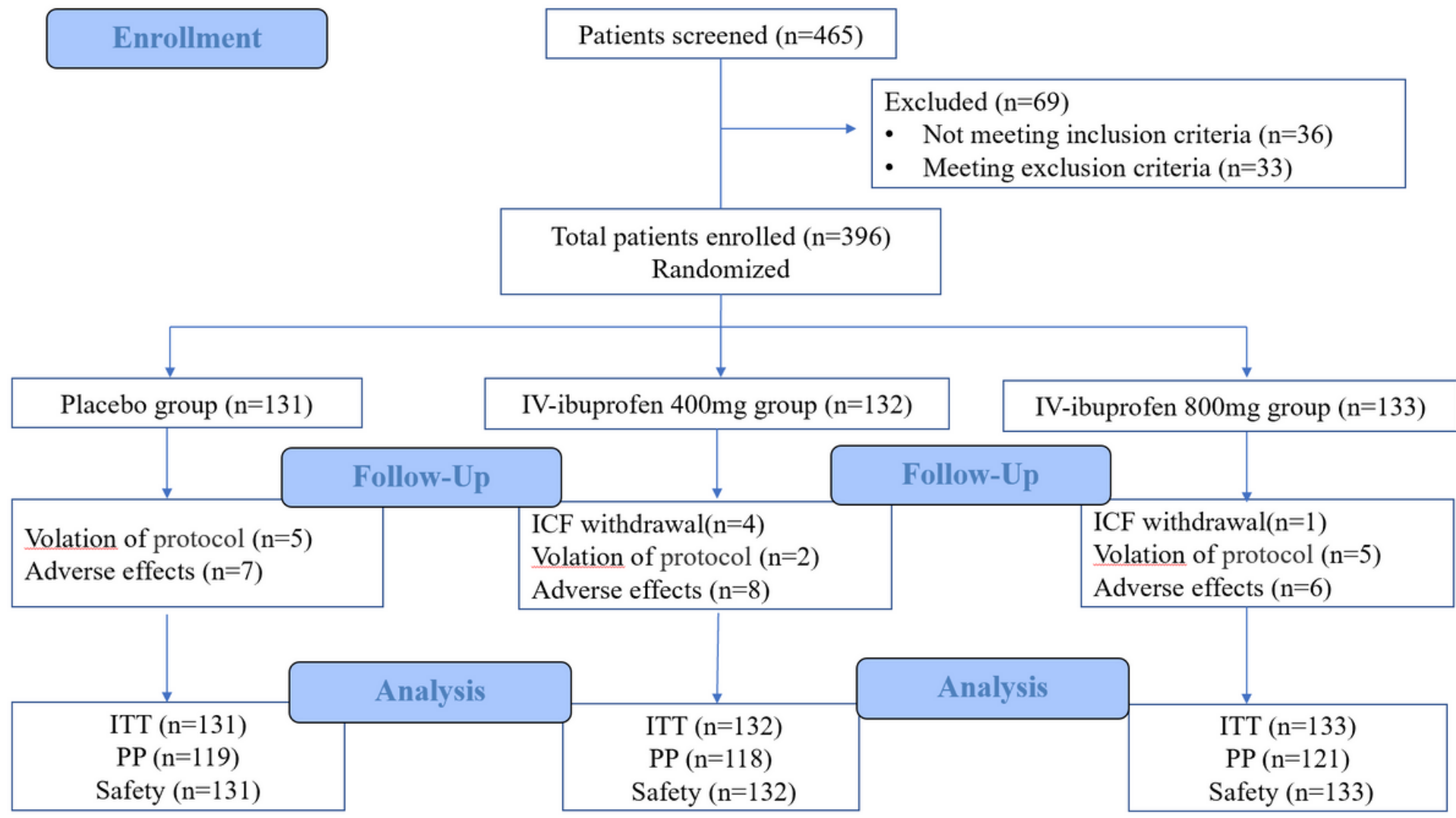

Figure 2 
Distribution of patients randomized to receive intravenous placebo, ibuprofen $400 \mathrm{mg}$, or ibuprofen $800 \mathrm{mg}$ for the management of postoperative pain. ICF: Informed Consent Form. ITT: intent-to-treat population, include all patients who were randomized and received at least a partial dose of IV ibuprofen or placebo. PP: per-protocol population, include all patients who completed 24 hours (at least 4 doses ibuprofen or placebo) of treatment according to the study protocol.

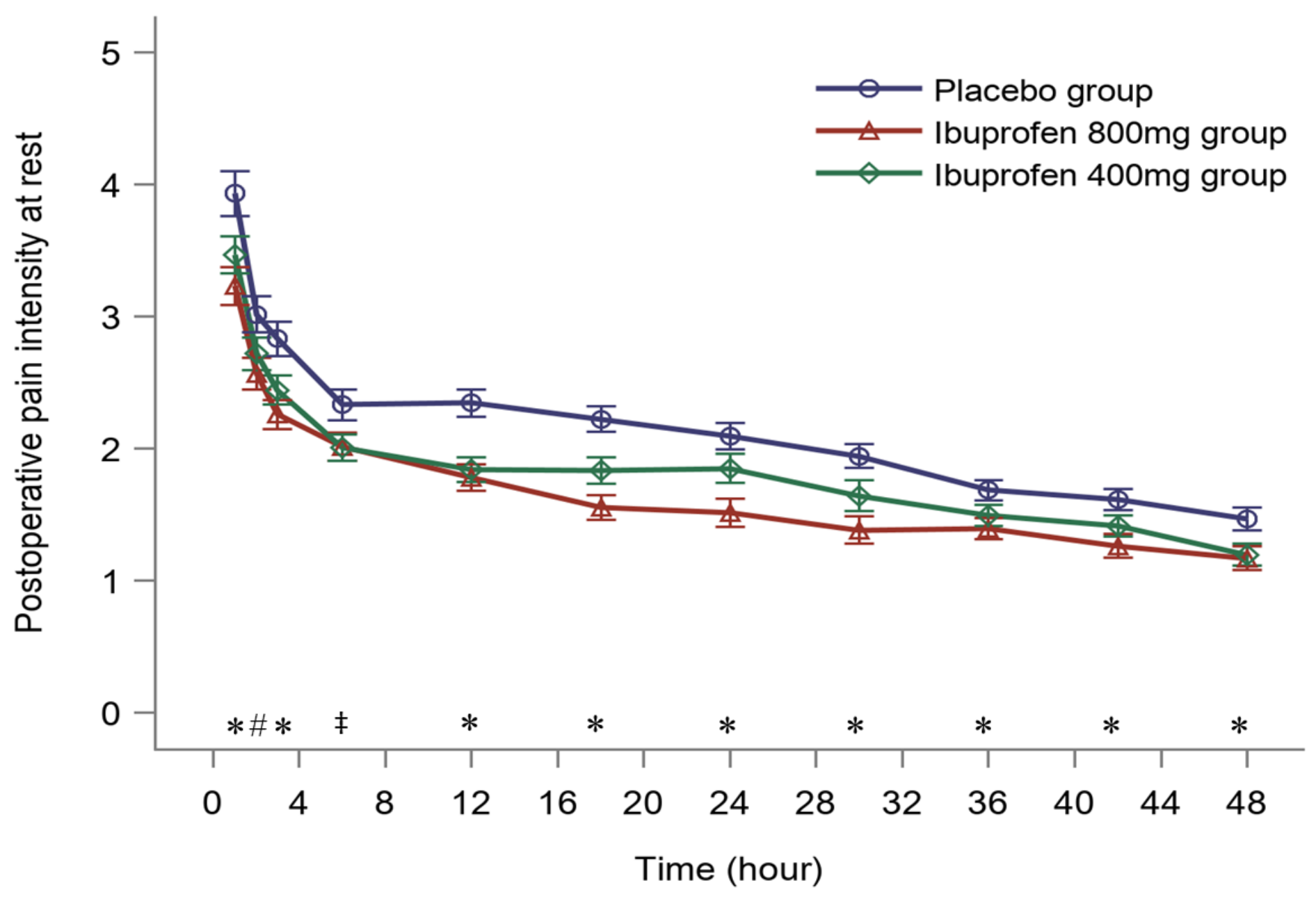

\section{Figure 3}

Postoperative Pain Intensity (PI) at rest. Patients self-reported pain scores with numerical rating scales (NRS) ( $0=$ no pain to $10=$ intense pain) at $0 \mathrm{~h}, 1 \mathrm{~h}, 2 \mathrm{~h}, 3 \mathrm{~h}, 6 \mathrm{~h}, 12 \mathrm{~h}, 18 \mathrm{~h}, 24 \mathrm{~h}, 30 \mathrm{~h}, 36 \mathrm{~h}, 42 \mathrm{~h}$ and $48 \mathrm{~h}$ immediately after

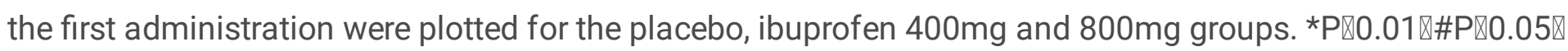
$\neq \mathrm{P}=0.05$. 


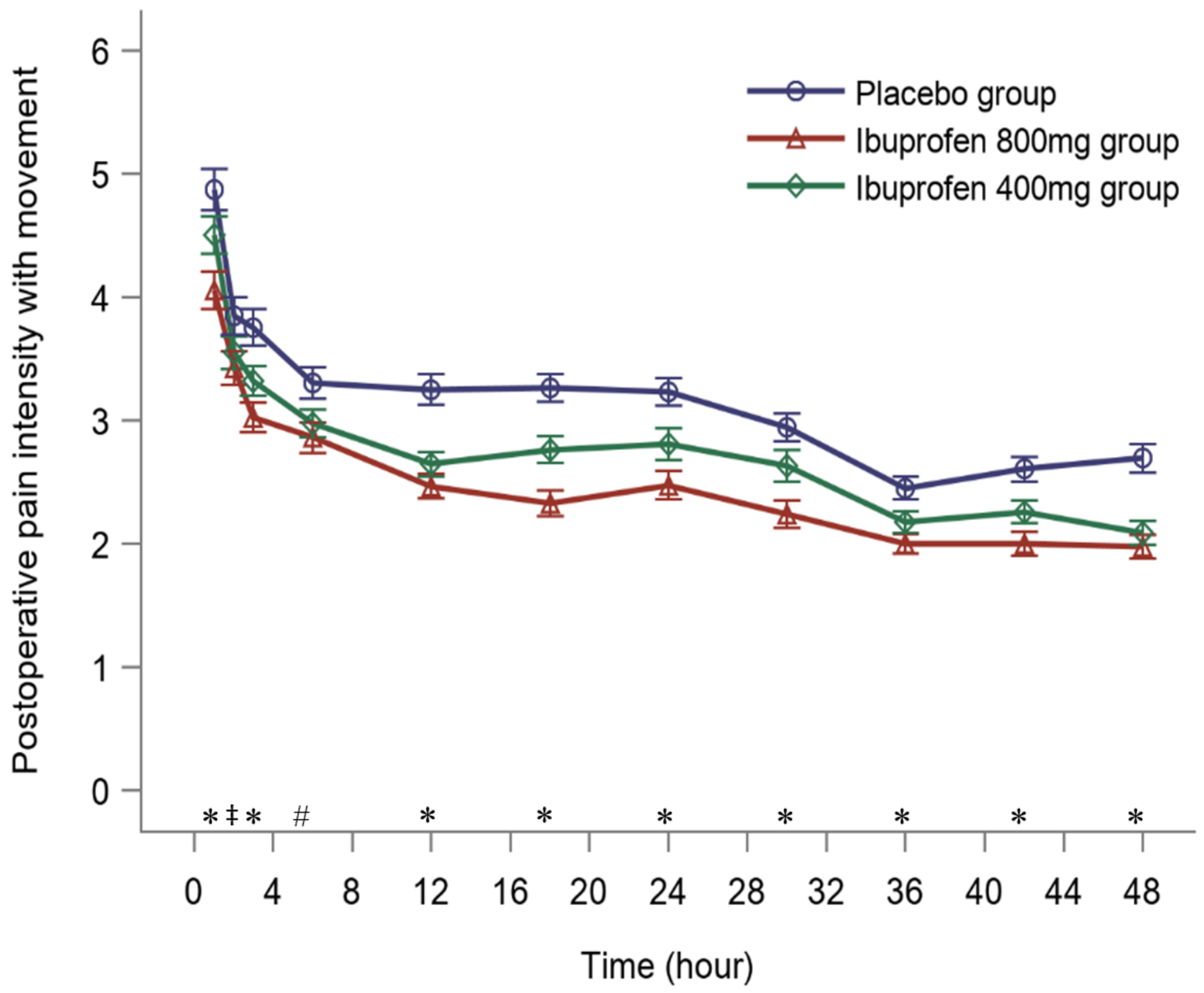

Figure 4

Postoperative Pain Intensity with movement. Patients self-reported pain scores with numerical rating scales (NRS) $(0=$ no pain to $10=$ intense pain) at $0 \mathrm{~h}, 1 \mathrm{~h}, 2 \mathrm{~h}, 3 \mathrm{~h}, 6 \mathrm{~h}, 12 \mathrm{~h}, 18 \mathrm{~h}, 24 \mathrm{~h}, 30 \mathrm{~h}, 36 \mathrm{~h}, 42 \mathrm{~h}$ and $48 \mathrm{~h}$ immediately after the first administration were plotted for the placebo, ibuprofen $400 \mathrm{mg}$ and $800 \mathrm{mg}$ groups. *P $\mathrm{*} 0.01 \rrbracket \# \mathrm{P} \otimes$ $0.05 \rrbracket \ddagger P=0.09$. 